\title{
Energy Dissipating Flows for Solving Nonlinear Eigenpair Problems
}

\author{
Ido Cohen and Guy Gilboa
}

June 5, 2018

\begin{abstract}
This work is concerned with computing nonlinear eigenpairs, which model solitary waves and various other physical phenomena. We aim at solving nonlinear eigenvalue problems of the general form $T(u)=\lambda Q(u)$. In our setting $T$ is a variational derivative of a convex functional (such as the Laplacian operator with respect to the Dirichlet energy), $Q$ is an arbitrary bounded nonlinear operator and $\lambda$ is an unknown (real) eigenvalue. We introduce a flow that numerically generates an eigenpair solution by its steady state.

Analysis for the general case is performed, showing a monotone decrease in the convex functional throughout the flow. When $T$ is the Laplacian operator, a complete discretized version is presented and analyzed. We implement our algorithm on Korteweg Vries $(\mathrm{KdV})$ and Nonlinear Schrdinger (NLS) equations in one and two dimensions. The proposed approach is very general and can be applied to a large variety of models. Moreover, it is highly robust to noise and to perturbations in the initial conditions, compared to classical Petiashvili-based methods.
\end{abstract}

\section{Introduction}

Nonlinear elliptic equations arise in various problems in physics, e.g. for stationary solutions of equations, such as Bose-Einstein condensates (BEC), Nonlinear Schrdinger (NLS) and Korteweg Vries (KdV) [38]. In this work we focus on nonlinear problems of the form,

$$
T(u)=\lambda Q(u),
$$

where $u$ is a function in a Banach space $\mathcal{U}$, and $T$ and $Q$ are (possibly) nonlinear operators. More specifically, we assume $T$ to be a subgradient of a convex, proper, lower-semi-continuous functional $J$,

$$
T(u) \in \partial_{u} J(u),
$$

where $\partial_{u} J(u)$ denotes the subdifferential of $J(u)$. On the right-hand-side of (1), $Q: \mathcal{U} \rightarrow \mathcal{U}$ is a bounded (possibly) nonlinear operator. We refer to functions $u$ 
which admit (1) as eigenfunctions, with a corresponding eigenvalue $\lambda \in \mathbb{R}$. Our aim is to find a pair $(u, \lambda) \in \mathcal{U} \times \mathbb{R}$ that admits (1), referred to as an eigenpair.

Eigenpairs of nonlinear operators appear in various fields of science and engineering. Their analysis can provide deeper understanding and significant insights related to nonlinear systems. Nonlinear eigenvalue analysis is an active field of research, from both a theoretical and a computational perspective. In some nonlinear problems, such as [17] the underlying operators are linear but the dependency on $\lambda$ is nonlinear. A recent review of such Nonlinear Eigenvalue Problems (NEP) appear in [22]. These studies are part of a different branch of problems (not in the scope of this paper). We examine solutions which can be formulated by Eq. (1), where the eigenvalue is linearly dependent. We summarize below the main related studies.

\subsection{Solitary Waves as Solutions of Nonlinear Eigenvalue Problems}

A pioneering work in this field, which was followed by many, is that of Petviashvili [33]. It was aimed at finding numerical approximations of stationary solutions for the Kadomtsev-Petviashvili equation with positive dispersion (KPI equation). The method, originally, was develop to obtain stationary solutions of wave equations of the form

$$
-M u+u^{p}=0,
$$

where $M$ is a positive, self-adjoint operator and $p$ is a constant. We note that $M$ should be invertible, as the iterative procedure is based on its inversion. Conditions for the convergence of Petviashvili's method were established in [31]. The approach of Petviashvili was later generalized and applied to a family of nonlinear problems, such as $[32,26,1,2]$. However, all these algorithms assume $M$ is invertible. Moreover, it is not aimed at finding eigenpairs, but at solving a more restricted problem. When casted within the formulation of (1), the eigenvalue is set to a unit value $(\lambda=1)$. Our proposed method is based on a forward flow, and hence $M$ is not require to be invertible. Moreover, it is aimed at finding eigenpairs, of unknown $\lambda$. The resulting eigenpair is related to an initial condition, provided by the user, which can emerge from noisy experimental data, for instance.

Yang and Lakoba $[26,40,25]$ generalized Petviashvili's iteration method, accelerated the inverse power method and used modified conjugate gradient to find solitary waves. In [39] it was suggested to combine the conjugate gradient method with accelerated inverse power method into a unified algorithm, which coincides with Petviashvili's method for small enough error. This method was shown to provide fast convergence rates. In our work we compare the numerical results to this method and to a modified version of it for adaptively computing eigenpairs. The focus of this paper is on the robustness of the methods, rather than on the convergence rate. We note that our forward flow requires considerably more iterations to converge, compared to algorithms based on inversion. However, it is much more stable and general. 


\subsection{Variational Formulations of Eigenpair Problems}

Eigenvalue problems are often analyzed theoretically and solved numerically based on energy minimization methods. Within a variational setting, it can be shown that an eigenpair is an extremum of a generalized Rayleigh quotient [37]. This extends in a natural manner the linear case, where any eigenvector $v$ of a Hermitian matrix $A$, admitting $A v=\lambda v$, is an extremal point of the associated Rayleigh quotient $R(v)=\left(v^{*} A v\right) /\left(v^{*} v\right)=\lambda$, where $v^{*}$ is the conjugate transpose of $v$. The studies of $[6,19,7,12]$ aim at finding a minimum (or a local minimum) of an energy functional associated to the eigenvalue problem. In $[6,19]$ a constrained steepest descent is used for solving ground states of BEC. Alternative approaches, such as [7, 12], are based on constrained energy minimization techniques with a suitable Lagrange function. In the above cases, both sides of the eigenvalue problem (1) should have an associated energy. This puts some limitations on the variety of problems that can be solved. In our work this restriction is relaxed (so only the operator $T$ is associated with an energy term).

\subsubsection{Eigenpairs Associated with Total Variation}

The total variation (TV) functional, $J_{T V}=\int|\nabla u(x)| d x$, has been thoroughly investigated in recent decades [11]. Since its introduction to the image processing field for denoising and deconvolution by [34], it has been used as an edge preserving regularizer for algorithms related to stereo, optical flow, segmentation and many other computer vision tasks [13]. Eigenpairs associated with TV were investigated by [27] and [3]. It was shown that convex disk-like shapes are eigenfunctions of the nonlinear eigenvalue problem

$$
T(u)=\lambda u, T(u) \in \partial_{u} J_{T V}(u)
$$

where $\partial_{u} J_{T V}(u)$ is the subdifferential of TV. For smooth, non-vanishing gradient, we have $\partial_{u} J_{T V}(u)=-\operatorname{div}(\nabla u /|\nabla u|)$, which is the 1-Laplacian operator. In recent years, a theory of nonlinear transforms for one-homogeneous functionals has been formulated $[20,21,10]$. It is based on the analysis of nonlinear eigenvalue problems. The work of [29] proposed a flow for finding eigenfunctions of one-homogeneous functionals, and is described in more detail below. Numerical methods for finding p-Laplacian eigenpairs were proposed in [41] and [24]. Solutions of semilinear elliptic eigen-problems were presented in [38]. Ground states of generalized eigenvalue problems, which may involve also a smoothing kernel, were analyzed in [8]. An iterative algorithm for finding nonlinear eigenpairs by an extended inverse-power method was proposed by [23]. All of these methods are based partially or mostly on the fact that the eigenpair satisfies an extremum of the associated (generalized) Rayleigh quotient.

Our work generalizes the flow of [29]. It goes beyond the variational setting and allows the nonlinear operator $Q$ in Eq. (1) to be very general. 


\subsection{Main Contributions}

The main contributions of this work are as follows:

1. A new flow is introduced, which generates eigenpairs admitting (1). A decrease in the convex functional $J$ associated with the operator $T$ is shown in the continuous setting, under mild assumptions (Sec. 2). When $J$ is a regularizing term, such as the Dirichlet energy, we get a dissipating flow which ensures robustness against noise and stability of the algorithm.

2. The need to omit residual parts perpendicular to the operator $T$ are explained. The concept of the $Q^{*}$ subspace, where all eigenfunctions must reside in, is introduced (Sec. 2.4). A complementary flow which directs the function $u$ to this subspace is presented and combined with the main flow (Sec. 2.5, Sec. 3).

3. A discrete explicit implementation is proposed and analyzed, when $T$ is the negative Laplacian. We formulate a time step bound that guarantees the monotonic decrease of $J$ throughout the flow (Section 4).

4. We employ our algorithm on KdV and NLS equation and draw a comparison between our algorithm and Newton Conjugate Gradient Methods (NCGM) [39] in one and two dimensions (section 5), showing excellent stability of the flow, for various initial conditions (Sec. 5).

\section{Flows for Generating Eigenpairs}

\subsection{Preliminary Notations and Definitions}

We first introduce some standard definitions and notations used in this paper. Let $\Omega$ be a bounded subset of $\mathbb{R}^{N}$. We use the $L^{2}$ inner product, $\langle u, v\rangle=$ $\int_{\Omega} u v \cdot d \mathbf{x}$ and its associated norm $\|u\|=\sqrt{\langle u, u\rangle}$. Let $J: \mathcal{U} \rightarrow \mathbb{R}$ be a convex functional. We denote by $\partial J(u)$ the subdifferential set of $J$ at $u$. Let us recall the subgradient inequality: For any $T \in \partial J(u)$, the following inequality holds:

$$
J(v) \geq J(u)+\langle T, v-u\rangle .
$$

The set of all $T$ for which this inequality holds is the subdifferential. A necessary and sufficient condition for $u$ to be a global minimum of $J$ is if zero is contained in $\partial J(u)$. We recall the "chain rule" for the differentiation of functionals (see Lemma 3.3 of Brezis [9] and recent extensions e.g. in [4]). Let $J$ be a convex, lower semi-continuous, proper functional, $\tau>0$, and $u \in W^{1,2}((0, \tau) ; \mathcal{U})$. Let also $T \in L^{2}((0, \tau) ; \mathcal{U})$, such that $T \in \partial J(u(t))$ a.e. in $(0, \tau)$. Then the function $J \circ u:[0, \tau] \rightarrow \mathbb{R}$ is absolutely continuous in $[0, \tau]$ with:

$$
\frac{d}{d t} J(u(t))=\left\langle T, u_{t}\right\rangle \quad \forall T \in \partial J(u(t)) \quad \text { a.e. in }(0, \tau) .
$$




\section{$2.2 \quad$ Nossek \& Gilboa Flow}

Our work generalizes the work of Nossek \& Gilboa [29], for which $J$ is assumed to be convex, absolutely one-homogeneous functional and $Q(u)=u$. We briefly describe this flow and its main properties. The flow is given by,

$$
u_{t}=\frac{u}{\|u\|}-\frac{T}{\|T\|}, \quad T \in \partial_{u} J, \quad u(0)=f,
$$

where $f$ admits $\|f\| \neq 0$ and has zero mean. If $J$ is invariant to a global constant change, i.e. $J(u)=J(u+c), \forall c \in \mathbb{R}$, the solution $u(t)$ of this flow has the following properties:

1. The mean value of $u(t)$ is preserved throughout the flow:

$$
\langle u(t), 1\rangle=0 .
$$

2. The value of $J(u(t))$ is decreasing with time

$$
\frac{d}{d t} J(u(t)) \leq 0, \text { a.e.in }(0, \infty)
$$

where equality is reached iff $u$ is an eigenfunction.

3. The $L^{2}$ norm of the solution is increasing with time,

$$
\frac{d}{d t}\|u(t)\|^{2} \geq 0
$$

where equality is reached iff $u$ is an eigenfunction.

4. A steady-state, $u_{t}=0$, holds iff $u$ is an eigenfunction.

For further details see [29]. Aujol et al. [5] analyzed the flow and proposed a modification for which existence and uniqueness of the continuous flow can be shown as well as convergence of the discrete iterative algorithm. Following this, we now turn to formulate the main flow which is designed to find solutions for Eq. (1). We will see later that an auxiliary (complementary) flow is required for this generalization to work properly, in order to allow minimal assumptions regarding the operator $Q$.

\subsection{The Main Flow}

We introduce a flow which generates eigenpairs of the form of Eq. (1). Given some initial condition $u(t=0)=f, u(t)$ is evolved by the following PDE,

$$
u_{t}(t)=M(u(t)),
$$

where

$$
M(u)=s \frac{Q(u)}{\|Q(u)\|}-\frac{T(u)}{\|T(u)\|},
$$

and $s=\operatorname{sign}(\langle Q(u), T(u)\rangle)$. 
Theorem 1. Assuming the flow of Eq. (7) exists and is unique and $J(u)$ satisfies Eq. (5), the solution $u(t)$ has the following attributes:

1. The value of $J$ is decreasing with time,

$$
\frac{d}{d t} J(u) \leq 0, \text { a.e. } \text { in }(0, \infty),
$$

where equality is reached iff $u$ admits Eq. (1).

2. A necessary condition for steady state $u_{t}=0$ holds iff $u$ is an eigenfunction.

Proof. We denote $T=T(u(t)), Q=Q(u(t))$.

1. From (5) and (7) we deduce,

$$
\begin{aligned}
\frac{d}{d t} J(u) & =\left\langle T, u_{t}\right\rangle \\
& =\left\langle T, s \frac{Q}{\|Q\|}-\frac{T}{\|T\|}\right\rangle \\
& =\frac{|\langle T, Q\rangle|}{\|Q\|}-\|T\| \leq 0, \quad \text { a.e.in }(0, \infty) .
\end{aligned}
$$

If $u$ is an eigenfunction in the sense of Eq. (1), then the eigenvalue $\lambda$ can be evaluated by using (1) and taking the inner-product with respect to either $Q$ or $T$, to have,

$$
\lambda=\frac{\langle T, Q\rangle}{\|Q\|^{2}}=\frac{\|T\|^{2}}{\langle T, Q\rangle}=\operatorname{sign}(\langle T, Q\rangle) \frac{\|T\|}{\|Q\|} .
$$

This yields $|\langle T, Q\rangle|=\|Q\| \cdot\|T\|$ and therefore $(d / d t) J(u)=0$. Conversely, if $(d / d t) J(u)=0$ then, following (8),

$$
|\langle T, Q\rangle|=\|T\| \cdot\|Q\|,
$$

i.e., $Q$ and $T$ are collinear and $u$ admits Eq. (1), hence an eigenfunction.

2. If $u$ admits Eq. (1) then we can assign $T=\lambda Q$ with $\lambda$ evaluated by the expression on the right of Eq. (9) to get $u_{t}=M(u)=0$ in (7). Conversely, let $u_{t}=0$ then $T$ and $Q$ are collinear, hence we reach Eq. (1).

\subsection{The Space of Solutions $\mathcal{Q}^{*}$}

Theorem 1 guarantees certain stability of the flow, since $J(u)$ decreases with time. In addition, the flow stops at an eigenpair, which is our aim. We will see in the analysis below that $u$ needs to be in a certain subspace of $\mathcal{U}$, otherwise, one will attain only trivial solutions of zero eigenvalue. We begin by stating some definitions and notations. Let us define two subsets of $\mathcal{U}, \mathcal{T}^{\perp}$ and $\mathcal{Q}^{*}$, associated with the operators $T$ and $Q$. 
Definition 1. Let $\mathcal{T}^{\perp}$ be the set perpendicular to the image of the operator $T$ :

$$
\mathcal{T}^{\perp}=\{v \in \mathcal{U}:\langle T(u), v\rangle=0, \forall u \in \mathcal{U}\} .
$$

We note that $\mathcal{T}^{\perp}$ is a linear subspace. This can be shown by taking two functions in that set $v_{1}, v_{2} \in \mathcal{T}^{\perp}$ and observing that the following relation holds:

$$
\left\langle T(u), \alpha_{1} v_{1}+\alpha_{2} v_{2}\right\rangle=\alpha_{1}\left\langle T(u), v_{1}\right\rangle+\alpha_{2}\left\langle T(u), v_{2}\right\rangle=0, \quad \forall u \in \mathcal{U}, \alpha_{1}, \alpha_{2} \in \mathbb{R} .
$$

We can now denote a basis for $\mathcal{T}^{\perp}$.

Definition 2. Let $\mathcal{B}$ be an orthonormal basis of $\mathcal{T}^{\perp}$.

Lemma 1. Let $\mathcal{T}^{\perp}$ be defined as in Def. 1, then:

1. $J(u)$ is invariant to addition of $v \in \mathcal{T}^{\perp}$, i.e.:

$$
J(u)=J(u+v), \quad \forall u \in \mathcal{U}, \forall v \in \mathcal{T}^{\perp} .
$$

2. If the following two conditions hold: (i) The operator $T$ is self-adjoint. (ii) For any minimizer $u$ of $J$, the subdifferential set, $\partial_{u} J(u)$, has a single element. Then $\mathcal{T}^{\perp}=\arg \min \{J(u)\}$.

Proof. 1. As $J(u)$ is convex then, based on the subdifferential inequality (4), we have

$$
J(u) \geq J(u+v)-\langle T(u+v), v\rangle=J(u+v), \quad \forall v \in \mathcal{T}^{\perp},
$$

and

$$
J(u+v) \geq J(u)+\langle T(u), v\rangle=J(u), \quad \forall v \in \mathcal{T}^{\perp} .
$$

2. If $u \in \arg \min \{J\}$ and $v \in \mathcal{T}^{\perp}$ then:

$$
\begin{aligned}
J(u) & \geq J(v)+\langle T(v),(u-v)\rangle \\
& =J(v)+\langle T(v), u\rangle \\
& =J(v)+\langle v, T(u)\rangle \\
& =J(v),
\end{aligned}
$$

but as $u$ minimizes $J$ we also have $J(v) \geq J(u)$, therefore $J(v)=J(u)$ and $v \in \arg \min \{J\}$. Conversely, if $u \in \arg \min \{J\}$ then $T(u)=0$, since $T$ is self-adjoint we get

$$
\langle T(u), v\rangle=\langle u, T(v)\rangle=0, \quad \forall v \in \mathcal{U} .
$$

Corollary 1. Let the conditions of Lemma 1, item 2 hold and $\min \{J(u)\}=0$, then: 
1. $\mathcal{T}^{\perp}$ is the null space of $J(u)$.

2. $J(u)$ is invariant to addition of elements in the null space.

We would now like to define a second significant subset, denoted by $\mathcal{Q}^{*}$.

Definition 3. Let $\mathcal{Q}^{*}$ be defined by

$$
\mathcal{Q}^{*}=\left\{u \in \mathcal{U}:\left\langle Q(u), e_{i}\right\rangle=0 \quad \forall e_{i} \in \mathcal{B}\right\},
$$

where $\mathcal{B}$ is defined in Def. 2 and $e_{i}$ are its orthonormal elements.

We would now like to define an energy which measures how far we are from $\mathcal{Q}^{*}$ :

$$
E(u)=\frac{1}{2} \sum_{e_{i} \in \mathcal{B}}\left\langle Q(u), e_{i}\right\rangle^{2} .
$$

Remark 1. An alternative definition of $\mathcal{Q}^{*}$ is the null space of the energy functional $E(u)$.

We observe that every $v \in \mathcal{T}^{\perp}$ is perpendicular to $Q\left(u^{*}\right)$ where $u^{*}$ is an eigenfunction with $\lambda \neq 0$. This is a straightforward consequence of Eq. (1) and Def. 1. Hence, $\lambda\left\langle Q\left(u^{*}\right), e_{i}\right\rangle=\left\langle T\left(u^{*}\right), e_{i}\right\rangle=0$. We can summarize this by the following statement:

Remark 2. All eigenfunctions with nonzero eigenvalue belong to $\mathcal{Q}^{*}$.

Proposition 1. Let $\mathcal{Q}^{*}$ be defined as in Def. 3 and $u(t)$ evolves according to $E q$. (7). If $u(t)$ is not an eigenfunction with $\lambda=0$, then the following properties hold for all $t \in(0, \infty)$ :

1. If $u(t) \notin \mathcal{Q}^{*}$ then $(d / d t) J(u(t))<0$.

2. If $u(t) \in \mathcal{Q}^{*}$ then the projection of $u(t)$ on the linear space $\mathcal{T}^{\perp}$ is preserved throughout the flow (Eq. (7)). In other words:

$$
\langle u(t), v\rangle=\text { const }, \quad \forall v \in \mathcal{T}^{\perp} .
$$

Proof. 1. This is an immediate consequence of Theorem 1 and Remark 2. According to Remark 2 and since we assume $u(t)$ is not an eigenfunction with zero eigenvalue, if $u(t) \notin \mathcal{Q}^{*}$ then $u(t)$ is necessarily not an eigenfunction. Recall Theorem 1, if $u(t)$ is not an eigenfunction then $(d / d t) J(u(t))<0$.

2. Let us compute the derivative of $\langle u(t), v\rangle$ with respect to time:

$$
\frac{d}{d t}\langle u(t), v\rangle=\left\langle u_{t}, v\right\rangle=\left\langle s \frac{Q(u)}{\|Q(u)\|}-\frac{T(u)}{\|T(u)\|}, v\right\rangle=s \frac{\langle Q(u), v\rangle}{\|Q(u)\|}-\frac{\langle T(u), v\rangle}{\|T(u)\|} .
$$

If $v \in \mathcal{T}^{\perp}$ then $\langle T(u), v\rangle=0$ and since $u \in \mathcal{Q}^{*}$ then $\langle Q(u), v\rangle=0$. Consequently,

$$
\frac{d}{d t}\langle u(t), v\rangle=0 \quad \Rightarrow \quad\langle u(t), v\rangle=\text { const. }
$$


Corollary 2. Let $T$ be a self adjoint operator and $\min \{J(u)\}=0$. If $u(t)$, the solution of the main flow Eq. (7), admits $u(t) \in \mathcal{Q}^{*}$, then its projection on the null space of $J$ is preserved.

This corollary is a generalization of the first attribute of the Nossek \& Gilboa flow which guarantees that the mean of $u$ is preserved. A more significant consequence of Prop. 1 is that if $u \notin \mathcal{Q}^{*}$ the main flow can reach a steady state only at "trivial" eigenfunctions, with $\lambda=0$. This leads us to propose a complementary flow which retains $u \in \mathcal{Q}^{*}$.

\subsection{Complementary flow}

The above discussion emphasizes the importance of retaining $u(t)$ to be in $\mathcal{Q}^{*}$. Following Remark 1 we define a complementary flow that aims at maintaining $E(u) \rightarrow 0$ without changing the value of $J(u)$. Let us first compute the variational derivative of $E(u)$ :

$$
\partial_{u} E=\sum_{i}\left\langle Q(u), e_{i}\right\rangle \cdot \partial Q_{i}(u),
$$

where

$$
\partial Q_{i}=\sum_{j}(-1)^{j} \frac{d^{j}}{d x^{j}}\left(\frac{\partial Q}{\partial u^{(j)}} e_{i}\right),
$$

and $u^{(j)}$ denotes the $j$ th derivative of $u$. We would like the complementary flow not to interfere with the main flow, and specifically not to increase $J$. A flow that decreases $E(u)$ on one hand and retains $J(u)$ unaffected on the other hand is:

$$
u_{t}=C(u)
$$

where

$$
C(u)=-\partial_{u} E+\frac{\left\langle\partial_{u} E, T(u)\right\rangle}{\|T(u)\|^{2}} T(u) .
$$

Let us compute the time derivatives of $J$ and $E$ :

$$
\begin{aligned}
\frac{d}{d t} J(u) & =\left\langle T(u), u_{t}\right\rangle=\langle T(u), C(u)\rangle \\
& =\left\langle T(u),-\partial_{u} E+\frac{\left\langle\partial_{u} E, T(u)\right\rangle}{\|T(u)\|^{2}} T(u)\right\rangle=0 .
\end{aligned}
$$

For $E$ we have,

$$
\begin{aligned}
\frac{d}{d t} E(u) & =\left\langle\partial_{u} E, u_{t}\right\rangle=\left\langle\partial_{u} E, C(u)\right\rangle \\
& =-\left\|\partial_{u} E\right\|^{2}+\frac{\left\langle\partial_{u} E, T(u)\right\rangle^{2}}{\|T(u)\|^{2}} \leq 0,
\end{aligned}
$$

where the last inequality follows Cauchy-Schwarz. Thus in the flow of (12) $E(u(t))$ decreases with time whereas the value of $J(u(t))$ is unchanged. Note that one should verify that $\partial_{u} E$ and $\partial_{u} J$ are not exactly parallel with opposite directions. The relations between $\partial_{u} E$ and $\partial_{u} J$ are farther discussed in Sec. 5 . 


\section{Combined Flows}

Constrained optimization problems in variational calculus are well studied, see e.g. $[18,16,15]$. In light of Prop. 1 we would like our flow (7) to be constrained to $u \in \mathcal{Q}^{*}$. Otherwise, we will either not reach a steady state or reach a trivial one (an eigenfunction of zero eigenvalue), which is usually not of interest. Such constrained flows are tied, though not equivalent, to exact penalty methods, as in $[16,15]$. Inspired by these works, we couple the main flow $(7)$ and the complementary flow (12) as follows,

$$
u_{t}=M(u)+\alpha C(u),
$$

where $\alpha \in \mathbb{R}_{+}$and $M(u)$ and $C(u)$ are as defined in the contexts of Eqs. (7) and (12), respectively. This combined flow should retain the properties of the main flow, while keeping $E(u)$ zero (for $\alpha$ large enough). Let us now show that $(d / d t) E(u)$ and $(d / d t) J(u)$ are nonpositive:

$$
\begin{aligned}
\frac{d}{d t} & J(u(t))=\left\langle T(u), u_{t}\right\rangle=\underbrace{\langle T(u), M(u)\rangle}_{\leq 0}+\alpha \underbrace{\langle T(u), C(u)\rangle}_{=0} \leq 0 . \\
\frac{d}{d t} E(u(t)) & =\left\langle\partial_{u} E, u_{t}\right\rangle=\left\langle\partial_{u} E, M(u)\right\rangle+\alpha\left\langle\partial_{u} E, C(u)\right\rangle \\
& =\left\langle\partial_{u} E, s \frac{Q(u)}{\|Q(u)\|}-\frac{T(u)}{\|T(u)\|}\right\rangle+\alpha\left(-\left\|\partial_{u} E\right\|^{2}+\frac{\left\langle\partial_{u} E, T(u)\right\rangle^{2}}{\|T(u)\|^{2}}\right) \\
& \leq\left\|\partial_{u} E\right\|-\frac{\left\langle\partial_{u} E, T(u)\right\rangle}{\|T(u)\|}+\alpha\left(-\left\|\partial_{u} E\right\|^{2}+\frac{\left\langle\partial_{u} E, T(u)\right\rangle^{2}}{\|T(u)\|^{2}}\right) \\
& =\underbrace{\left(-\left\|\partial_{u} E\right\|+\frac{\left\langle\partial_{u} E, T(u)\right\rangle}{\|T(u)\|}\right)}_{\leq 0}\left(-1+\alpha\left(\left\|\partial_{u} E\right\|+\frac{\left\langle\partial_{u} E, T(u)\right\rangle}{\|T(u)\|}\right)\right) .
\end{aligned}
$$

The inequality of the third row is based on Cauchy-Schwarz inequality, $s\left\langle\partial_{u} E, Q(u)\right\rangle /\|Q(u)\| \leq$ $\left\|\partial_{u} E\right\|$. If $\alpha \gg 1$ and the elements $\partial_{u} E(u)$ and $T(u)$ are not collinear (in opposite directions) we can deduce that:

$$
\frac{d}{d t} E(u(t)) \leq 0 .
$$

\subsection{Towards a Discrete Setting}

We propose in Algorithm 1 the general discrete iterations that approximate the combined flow of (15). It is based on an explicit scheme for the time discretization. The time step parameters $d t_{C}$ and $d t_{M}$ are not resolved here. A full analysis and bounds on these time steps is given next, for the common case of $T(u)=-\Delta u$.

Here, instead of choosing a specific $\alpha$, the complementary flow is evolved (for each time step of the main flow) until a certain accuracy is reached, with respect 
to being close enough to $\mathcal{Q}^{*}$. This is analogous to having a time dependent $\alpha(t)$ in (15). An illustration of the combined flow is shown in Fig. 1a where $M(u)$ and $C(u)$ are invoked alternatingly. As discussed above, in the continuous setting $C(u)$ does not change the value of the functional $J(u)$ and is along its level lines. However, $M(u)$ might cause $u$ to be out of $\mathcal{Q}^{*}$. The complementary flow, $C(u)$, "returns" $u$ to $\mathcal{Q}^{*}$ leaving $J(u)$ unchanged. Note that in the exact penalty algorithm [16] the flow is run in several iterations with increasing $\alpha$. The combined flow is repeated until the solution is close enough to an eigenpair in terms of the respective angle (See Fig. 1b and Algo. 1).

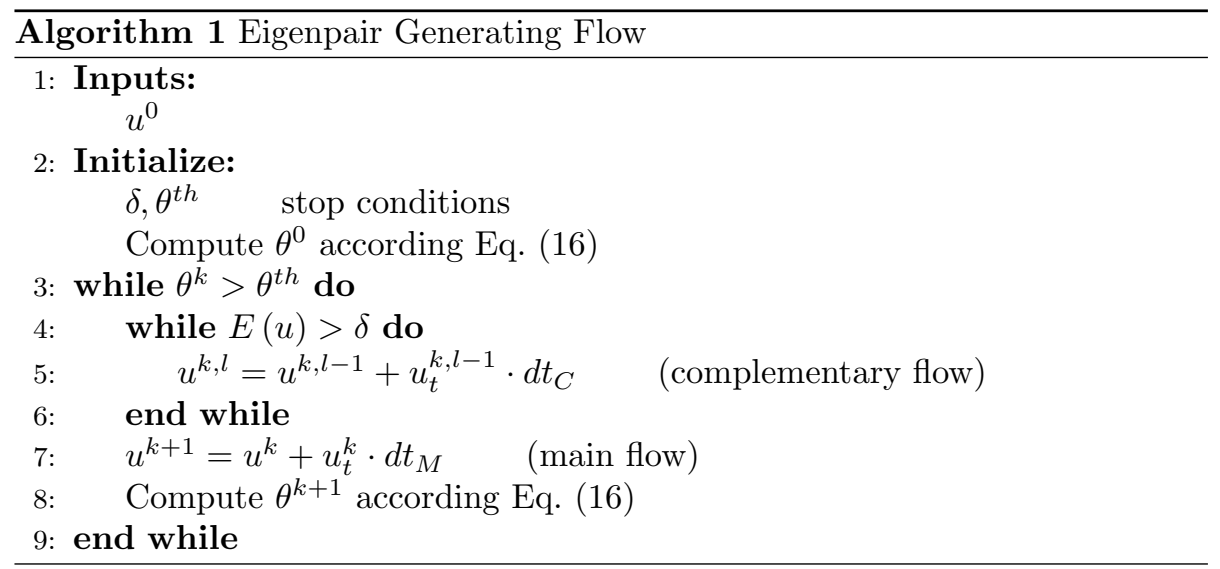

Our stopping condition is based on the absolute angle between $T(u)$ and $Q(u)$,

$$
|\cos (\theta)|=\frac{|\langle T(u), Q(u)\rangle|}{\|T(u)\| \cdot\|Q(u)\|} .
$$

When the angle $\theta$ is zero, $T$ and $Q$ are collinear, and an exact eigenpair is

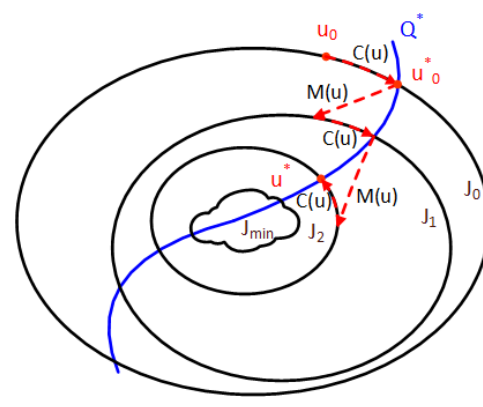

(a) $J$ functional contour

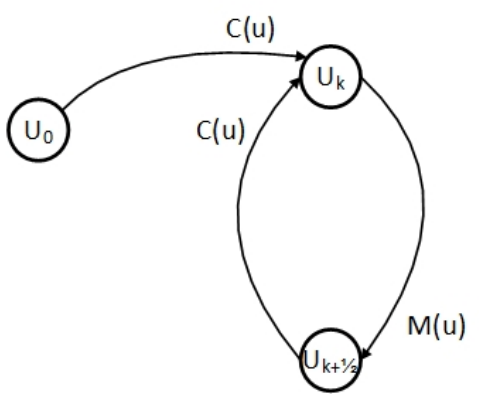

(b) The combined flow as finite state machine

Figure 1: An illustration of the infinitesimally approximation of the combined flow. 
attained. For numerical purposes, we seek a good approximation and stop when $\theta$ is below a certain threshold. Further discussions related to the stopping condition are in $\mathrm{A}$.

\subsection{Realization for $T(u)=-\Delta u$}

As many phenomena are based on eigenpairs, in the sense of Eq. (1), with $T(u)$ the negative Laplacian operator, we devote the rest of this paper to that particular case. In this case $J$ is the Dirichlet energy,

$$
J(u)=\frac{1}{2}\|\nabla u\|^{2} .
$$

It is clear that $J$ admits the conditions of $E q$. (1) and $E q$. (5). The null space of the Dirichlet energy is all functions in $\mathcal{U}$ of constant value. According to Cor. 2 it coincides with $\mathcal{T}^{\perp}$. Therefore,

$$
\mathcal{T}^{\perp}=\{v \in U: v=\text { const }\} .
$$

The orthonormal basis of $\mathcal{T}^{\perp}$ is

$$
\mathcal{B}=\left\{\frac{1}{\sqrt{|\Omega|}}\right\} .
$$

The energy functional $E(u)$ is defined by,

$$
\begin{gathered}
E(u)=\frac{1}{2|\Omega|}\langle Q(u), 1\rangle^{2}, \\
\partial E=\frac{1}{|\Omega|}\langle Q(u), 1\rangle \partial Q,
\end{gathered}
$$

and $\partial Q$ is the variational derivative of $\langle Q(u), 1\rangle$. Naturally, $\mathcal{Q}^{*}$ is all $u \in \mathcal{U}$ such that $\int_{\Omega} Q(u) d \mathbf{x}=0$. The main and complementary operators, respectively, are,

$$
M(u)=s \frac{Q(u)}{\|Q(u)\|}+\frac{\Delta u}{\|\Delta u\|}
$$

where $s=\operatorname{sign}(\langle Q(u),-\Delta u\rangle)$, and

$$
C(u)=-\partial_{u} E+\frac{\left\langle\partial_{u} E, \Delta u\right\rangle}{\|\Delta u\|^{2}} \Delta u .
$$

In the following section we resolve the time step sizes for the discrete implementation when $T$ is the negative Laplacian operator.

\section{Implementation for the Laplacian operator}

For the discrete implementation of the flow we follow the general algorithm presented in the previous section. However, instead of performing a full iterative 
process of the complementary flow for each main time step, we perform a single step. It is shown that for $T=-\Delta$, with an appropriate time step of the complementary flow, the energy $E$ vanishes, up to a first order Taylor approximation. We suggest the following iterations to approximate the combined flow: Initialize with $u^{0}$, iterate until convergence,

$$
\begin{aligned}
& u^{k+\frac{1}{2}}=u^{k}+M\left(u^{k}\right) \cdot d t_{M} \\
& u^{k+1}=u^{k+\frac{1}{2}}+C\left(u^{k+\frac{1}{2}}\right) \cdot d t_{C} .
\end{aligned}
$$

In the rest of this section we set bounds for $d t_{M}$ and $d t_{C}$, to ensure stable flows. These bounds guarantee that in every cycle (from $k$ to $k+1$ ) we obtain $J\left(u^{k}\right)-J\left(u^{k+1}\right) \leq 0$ and $E\left(u^{k}\right) \cong 0, \quad \forall k$.

\subsection{Characterization of $J(u)$ and $E(u)$ within the Discrete Setting}

We now proceed with analyzing the behavior of $J\left(u^{k}\right)$ and $E\left(u^{k}\right)$ using the iterations of (19), where $M\left(u^{k}\right)$ and $C\left(u^{k}\right)$ are defined by (17) and (18). For simplicity, we use the same notations of gradient $(\nabla)$ and Laplacian $(\Delta)$ for the discrete operators.

\subsubsection{Main Flow Iteration}

We first analyze the influence of an iteration of the main flow (first row of Eq. (19)) on the functional $J$. We use the standard first order backward difference time-step approximation of explicit schemes. We examine the change in $J$ at iteration $k$, denoted by $D_{J}^{k}$, following a time step of the main flow:

$$
\begin{aligned}
D_{J}^{k} & :=J\left(u^{k+\frac{1}{2}}\right)-J\left(u^{k}\right) \\
& =J\left(u^{k}+d t_{M} \cdot M\left(u^{k}\right)\right)-J\left(u^{k}\right) .
\end{aligned}
$$

Using the identity $J(u)=\frac{1}{2}\langle-\Delta u, u\rangle$ yields:

$$
\begin{aligned}
D_{J}^{k} & =\frac{1}{2}\left(\left\langle-\Delta\left(u^{k}+d t_{M} \cdot M\left(u^{k}\right)\right), u^{k}+d t_{M} \cdot M\left(u^{k}\right)\right\rangle-\left\langle-\Delta u^{k}, u^{k}\right\rangle\right) \\
& =d t_{M}\left\langle-\Delta u^{k}, M\left(u^{k}\right)\right\rangle+\frac{1}{2} d t_{M}^{2}\left\|\nabla M\left(u^{k}\right)\right\|^{2} .
\end{aligned}
$$

According to Theorem 1 the expression $\left\langle\Delta u^{k}, M\left(u^{k}\right)\right\rangle$ is non-negative, thus, if

$$
0 \leq d t_{M} \leq 2 \frac{\left\langle\Delta u^{k}, M\left(u^{k}\right)\right\rangle}{\left\|\nabla M\left(u^{k}\right)\right\|^{2}}
$$

then $D_{J}^{k} \leq 0$.

Let us now examine the influence of the main flow on the functional $E$. We assume $E\left(u^{k}\right)$ is approximately zero (up to a first order Taylor approximation), 
to be justified in the next paragraph. The Taylor approximation of $E\left(u^{k+\frac{1}{2}}\right)$ is given by:

$$
\begin{aligned}
E\left(u^{k+\frac{1}{2}}\right) & =\frac{1}{2|\Omega|}\left\langle Q\left(u^{k+\frac{1}{2}}\right), 1\right\rangle^{2} \\
& =\frac{1}{2|\Omega|}\left\langle Q\left(u^{k}+d t_{M} M\left(u^{k}\right)\right), 1\right\rangle^{2} \\
& =\frac{1}{2|\Omega|}\left(\left\langle Q\left(u^{k}\right), 1\right\rangle+d t_{M}\left\langle\partial Q\left(u^{k}\right), M\left(u^{k}\right)\right\rangle+O\left(d t_{M}^{2}\right)\right)^{2} \\
& \cong \frac{1}{2|\Omega|}\left(\left\langle Q\left(u^{k}\right), 1\right\rangle+d t_{M}\left\langle\partial Q\left(u^{k}\right), M\left(u^{k}\right)\right\rangle\right)^{2}
\end{aligned}
$$

Under the assumption $E\left(u^{k}\right)$ is approximately zero (i.e. $\left\langle Q\left(u^{k}\right), 1\right\rangle \cong 0$ ) we have,

$$
\begin{aligned}
D_{E}^{k} & :=E\left(u^{k+\frac{1}{2}}\right)-E\left(u^{k}\right) \\
& \cong E\left(u^{k+\frac{1}{2}}\right) \\
& \cong d t_{M}^{2} \cdot \frac{\left\langle\partial Q\left(u^{k}\right), M\left(u^{k}\right)\right\rangle^{2}}{2|\Omega|} .
\end{aligned}
$$

\subsubsection{Complementary flow iteration}

We now analyze the influence of the complementary flow (second row of Eq. (19)) on the energies $J$ and $E$. Here we begin with the analysis of $E(u)$.

$$
\begin{aligned}
D_{E}^{k+\frac{1}{2}} & :=E\left(u^{k+1}\right)-E\left(u^{k+\frac{1}{2}}\right) \\
& =E\left(u^{k+\frac{1}{2}}\right)+d t_{C} \cdot\left\langle\partial E\left(u^{k+\frac{1}{2}}\right), C\left(u^{k+\frac{1}{2}}\right)\right\rangle+O\left(d t_{C}^{2}\right)-E\left(u^{k+\frac{1}{2}}\right) \\
& \cong d t_{C} \cdot\left\langle\partial E\left(u^{k+\frac{1}{2}}\right), C\left(u^{k+\frac{1}{2}}\right)\right\rangle .
\end{aligned}
$$

The step size for which $E\left(u^{k+1}\right)$ vanishes, as a first order approximation, is therefore

$$
d t_{C}=-\frac{E\left(u^{k+\frac{1}{2}}\right)}{\left\langle\partial E\left(u^{k+\frac{1}{2}}\right), C\left(u^{k+\frac{1}{2}}\right)\right\rangle} .
$$

The step size $d t_{C}$ is positive since the denominator is negative (according to Eq. (14)).

Finally, we examine the influence of the complementary flow on the functional $J$. The change of $J$ along one time step of the complementary flow is

$$
\begin{aligned}
D_{J}^{k+\frac{1}{2}} & :=J\left(u^{k+1}\right)-J\left(u^{k+\frac{1}{2}}\right) \\
& =\frac{1}{2}\left(\left\langle-\Delta u^{k+1}, u^{k+1}\right\rangle-\left\langle-\Delta u^{k+\frac{1}{2}}, u^{k+\frac{1}{2}}\right\rangle\right) \\
& =\frac{1}{2}\left(\left\langle-\Delta\left(u^{k+\frac{1}{2}}+d t_{C} \cdot C\left(u^{k+\frac{1}{2}}\right)\right), u^{k+\frac{1}{2}}+d t_{C} \cdot C\left(u^{k+\frac{1}{2}}\right)\right\rangle-\left\langle-\Delta u^{k+\frac{1}{2}}, u^{k+\frac{1}{2}}\right\rangle\right) .
\end{aligned}
$$


Note that according to Eq. (13) $\left\langle\Delta u^{k+\frac{1}{2}}, C\left(u^{k+\frac{1}{2}}\right)\right\rangle=0$ and therefore

$$
D_{J}^{k+\frac{1}{2}}=d t_{C}^{2}\left\|\nabla C\left(u^{k+\frac{1}{2}}\right)\right\|^{2}
$$

Using Eq. (23),

$$
D_{J}^{k+\frac{1}{2}}=E^{2}\left(u^{k+\frac{1}{2}}\right) \frac{\left\|\nabla C\left(u^{k+\frac{1}{2}}\right)\right\|^{2}}{\left\langle\partial E\left(u^{k+\frac{1}{2}}\right), C\left(u^{k+\frac{1}{2}}\right)\right\rangle^{2}} .
$$

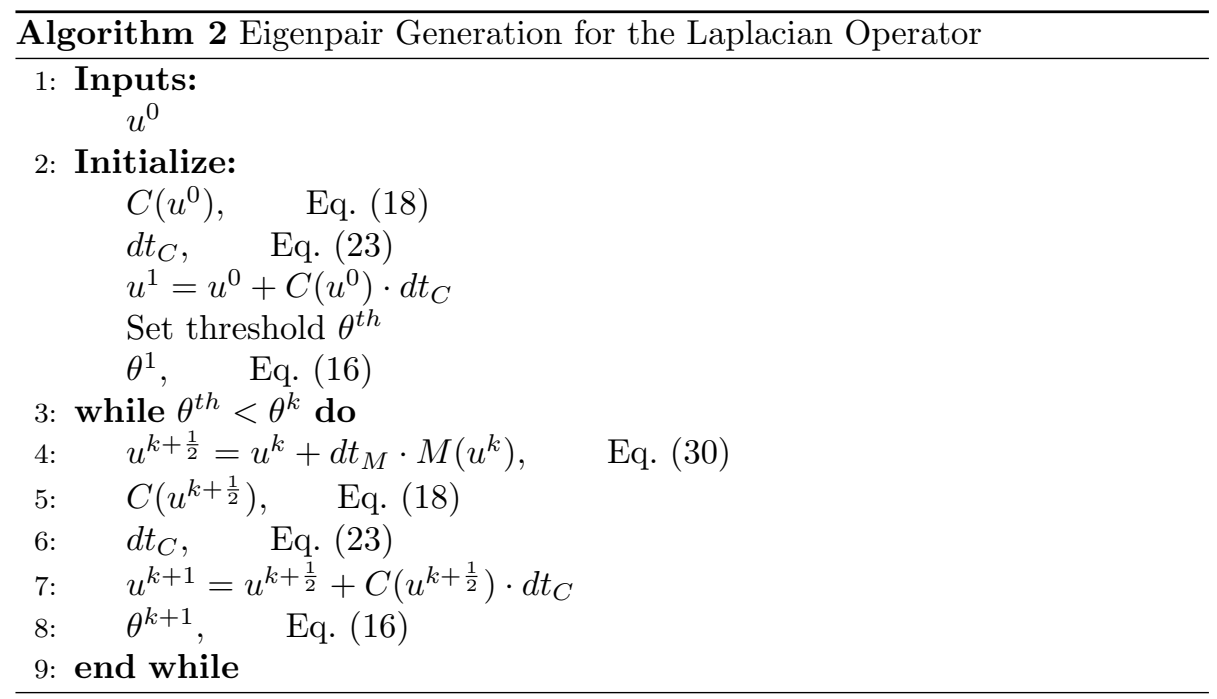

\subsubsection{Conditions for the monotonic decrease of $J$}

Following the above constraints on the time step sizes of both flows we can now analyze the change in $J$ for the entire flow, Eq. (19),

$$
J\left(u^{k+1}\right)-J\left(u^{k}\right)=D_{J}^{k+\frac{1}{2}}+D_{J}^{k} .
$$

We would like to find a bound on $d t_{M}$ which ensures $J\left(u^{k+1}\right)-J\left(u^{k}\right) \leq 0$. Substituting for $D_{J}^{k+\frac{1}{2}}$ and $D_{J}^{k}$ by Eqs. (24) and (20), respectively, we have,

$$
\begin{aligned}
J\left(u^{k+1}\right)-J\left(u^{k}\right)= & E^{2}\left(u^{k+\frac{1}{2}}\right) \frac{\left\|\nabla C\left(u^{k+\frac{1}{2}}\right)\right\|^{2}}{\left\langle\partial E\left(u^{k+\frac{1}{2}}\right), C\left(u^{k+\frac{1}{2}}\right)\right\rangle^{2}}+ \\
& d t_{M}\left\langle-\Delta u^{k}, M\left(u^{k}\right)\right\rangle+\frac{1}{2} d t_{M}^{2}\left\|\nabla M\left(u^{k}\right)\right\|^{2} .
\end{aligned}
$$


The term $E\left(u^{k+\frac{1}{2}}\right)$ is evaluated according to the first order approximation of Eq. (22) to obtain,

$$
\begin{aligned}
J\left(u^{k+1}\right)-J\left(u^{k}\right) \cong & d t_{M}^{4} \cdot \frac{\left\langle\partial Q\left(u^{k}\right), M\left(u^{k}\right)\right\rangle^{4}}{4|\Omega|^{2}} \cdot \frac{\left\|\nabla C\left(u^{k+\frac{1}{2}}\right)\right\|^{2}}{\left\langle\partial E\left(u^{k+\frac{1}{2}}\right), C\left(u^{k+\frac{1}{2}}\right)\right\rangle^{2}}+ \\
& d t_{M}\left\langle-\Delta u^{k}, M\left(u^{k}\right)\right\rangle+\frac{1}{2} d t_{M}^{2}\left\|\nabla M\left(u^{k}\right)\right\|^{2} .
\end{aligned}
$$

A deeper analysis shows that when the directions of $\partial E(u)$ and $\Delta u$ are far enough from being collinear the fourth order term is small and thus can be omitted. After neglecting the fourth order term on the right-hand-side of Eq. (26) the inequality coincides with Eq. (21). We now use the following inequality based on the operator norm of the discrete gradient:

$$
\|\nabla u\|^{2} \leq \frac{4 d}{h^{2}}\|u\|^{2},
$$

where $d$ is the dimension and $h$ is the spatial grid size. Based on the expression on the right-hand-side of Eq. (21), and the definition of $M(u)$ for the Laplacian case, (17), we have

$$
2 \frac{\left\langle\Delta u^{k}, M\left(u^{k}\right)\right\rangle}{\left\|\nabla M\left(u^{k}\right)\right\|^{2}} \geq \frac{h^{2}}{2 d} \frac{\left\langle\Delta u^{k}, M\left(u^{k}\right)\right\rangle}{\left\|M\left(u^{k}\right)\right\|^{2}}=\frac{h^{2}}{2 d} \frac{\frac{\left|\left\langle\Delta u^{k}, Q\left(u^{k}\right)\right\rangle\right|}{\left\|Q\left(u^{k}\right)\right\|}+\left\|\Delta u^{k}\right\|}{2+2 \frac{\left|\left\langle\Delta u^{k}, Q\left(u^{k}\right)\right\rangle\right|}{\left\|\Delta u^{k}\right\| \cdot\left\|Q\left(u^{k}\right)\right\|}}=\frac{h^{2}}{4 d}\left\|\Delta u^{k}\right\| .
$$

Therefore, to ensure the expression in (26) is negative, we set the following bound on the time step size,

$$
d t_{M} \leq \frac{h^{2}}{4 d}\left\|\Delta u^{k}\right\| .
$$

According to Eq. (20), $D_{J}^{k}$ depends on $d t_{M}$ quadratically, therefore, the optimal $d t_{M}$ is half of the upper bound. Consequently, we choose

$$
d t_{M}=\frac{h^{2}}{8 d}\left\|\Delta u^{k}\right\|
$$

Typical values of $d t_{M}$ in our experiments were in the range $[0.002,0.6]$ for one dimension and $[0.05,0.13]$ for two dimensions. These values highly depend on the noise level. A detailed description of the algorithm, in the case of the Laplacian operator, is presented in Algorithm 2.

\subsection{Interpretations}

Let us revisit Eq. (1) and the main flow. Given $u^{k}$ we can evaluate the eigenvalue according to Eq. (9) and define the error of the solution $u^{k}$ as:

$$
\operatorname{err}\left(u^{k}\right)=-\Delta u^{k}-\lambda\left(u^{k}\right) \cdot Q\left(u^{k}\right)
$$


which is zero if $u^{k}$ is an eigenfunction. In addition, by using the expression of $d t_{M}$ in Eq. (30), we can calculate the change of $u$ at the $k$ th step as,

$$
\begin{aligned}
u^{k+\frac{1}{2}}-u^{k} & =M\left(u^{k}\right) \cdot d t_{M} \\
& =\frac{h^{2}}{8 d}\left(\Delta u^{k}+\operatorname{sign}\left(\left\langle Q\left(u^{k}\right),-\Delta u^{k}\right\rangle\right) \frac{\left\|\Delta u^{k}\right\|}{\left\|Q\left(u^{k}\right)\right\|} Q\left(u^{k}\right)\right) \\
& =\frac{h^{2}}{8 d}\left(\Delta u^{k}+\lambda\left(u^{k}\right) \cdot Q\left(u^{k}\right)\right) \\
& =-\frac{h^{2}}{8 d} \cdot \operatorname{err}\left(u^{k}\right) .
\end{aligned}
$$

We note that [26] suggests a general eigenvalue approximation by: $\lambda(u)=$ $\langle-\Delta u, f(u)\rangle /\langle Q(u), f(u)\rangle$ with an arbitrary operator $f(u)$ as long as this ratio exists. In particular we can have $f(u)=u$. Then we can replace Line 4 in Algo. 2 with Eq. (32) using other eigenvalue evaluations. We can conclude that any flow in the sense of Eq. (32) with an eigenvalue approximation for which Theorem 1 holds, the later properties of Sec. 2 (Lemma 1 and Prop. 1) also hold. This conclusion is valid not only for $T(u)=-\Delta u$ but for any operator $T(u)$ admitting Eq. (1). However, different step size constraints should be found.

\section{Applications}

In this section we present several numerical experiments using the proposed method. We compare our results with NCGM introduced in [39]. NCGM assumes the eigenvalue is given, therefore we supply the correct eigenvalue to the algorithm. In addition, we compare our method to an adaptive version of NCGM when the eigenvalue is not given (thus the task is to find a full eigenpair, as our flow does). The eigenvalue is approximated at each iteration based on [26]. We denote this method by Adaptive NCGM (ANCGM).

\subsection{Dimension}

\subsubsection{Soliton Equations}

In 1895 Korteweg Vries (KdV) formulated a mathematical model of waves on shallow water surfaces which were previously described by Russell. Later studies have shown that the $\mathrm{KdV}$ equation is the continuum limit of a discrete nonlinear mass-spring model [30]. The formulation by Kruskal and Zabusky [42] for such phenomena was:

$$
u_{t}+u u_{x}+\delta^{2} u_{x x x}=0,
$$

with $\delta$ a small real scalar. Reformulating this expression for a stationary wave yields the following equation:

$$
-u_{X X}=\lambda\left(-c u+\frac{u^{2}}{2}\right),
$$


where $c$ is the wave velocity, $X=x-c t$ and $\lambda=\delta^{-2}$. Naturally, $\lambda$ can be understood as an eigenvalue. In this example we have $Q(u)=-c u+u^{2} / 2$. The solution to this equation models well a family of solitary waves referred to as solitons, (named by Kruskal and Zabusky). In this case an analytic solution can be formulated, which makes it feasible to compare the experimental results to the ground truth. The solution for (33) is:

$$
u(X)=3 c \cdot \operatorname{sech}^{2}\left(\frac{\sqrt{c \cdot \lambda} X}{2}\right) .
$$

The implementation of our flow is based on Algo. 2, where for solving Eq. (33) we have:

$$
\begin{aligned}
Q(u) & =-c u+\frac{u^{2}}{2}, \\
\partial Q(u) & =-c+u, \\
\partial_{u} E & =\langle Q(u), 1\rangle(-c+u) .
\end{aligned}
$$

The operators in NCGM [39] are:

$$
\begin{aligned}
L & =\Delta+\lambda(c-u), \\
M & =\Delta+\lambda \cdot c,
\end{aligned}
$$

where $\lambda$ is given. The notations $L$ and $M$ are taken from [39] (not to confuse with $M(u)$ of our algorithm). In the adaptive version, ANCGM, $\lambda$ is approximated by $\lambda(u)=\langle-\Delta u, u\rangle /\langle Q(u), u\rangle$.

\subsubsection{Experimental setup}

In our experiments we initialize with the a noisy version of the solution, by adding white Gaussian noise $N$ of a certain variance to the analytic solution for some given $\lambda$. Note that we also allow some bias in the simulated measurements so the noise mean in not zero. The noise parameters are denoted by a normal distribution $N \sim \mathcal{N}\left(\mu, \sigma^{2}\right)$, with mean $\mu$ and variance $\sigma^{2}$. We employ the aforementioned algorithms with a stopping condition $\theta^{t h} \leq 2^{\circ}$ (where $\theta$ is the angle between $T$ and $Q$, as defined in (16)).

In Fig. 2 the results of the generated eigenfunctions are shown. The noise parameters are $\mu=0.1$ with variance values of $\sigma^{2}=0.2$ and $\sigma^{2}=0.36$, for the first and second experiments, respectively. For this relatively simple case we compare our results to ANCGM, where both algorithms try to find an eigenpair ( $\lambda$ is not known). We show that our algorithm is quite stable, for different levels of noise. ANCGM on the other hand, collapses to the trivial solution $(-\Delta u=$ $\lambda Q(u)=0$ ) for high noise levels (and is less accurate also for moderate noise). We should emphasize that our algorithm runs considerably more iterations than ANCGM. However, our iterations are very fast, as they are based on explicit forward-flow computations, compared to computationally-intensive iterations of 


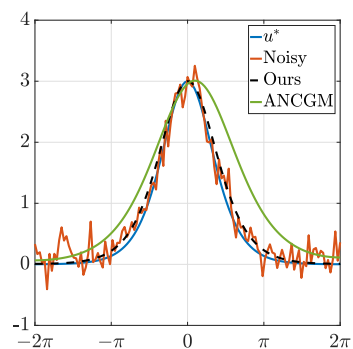

(a) $N \sim \mathcal{N}(0.1,0.2)$

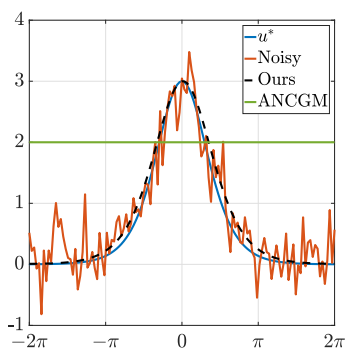

(b) $N \sim \mathcal{N}(0.1,0.36)$

Figure 2: A comparison of eigenpair generating algorithms for $\mathrm{KdV}$ equation with different noise levels. In Fig. 2a we summarize the results of ANCGM and our flow when the initial function $u^{0}$, red solid line, is an eigenfunction with additive white Gaussian noise (of mean of 0.1 and variance of 0.2 ). In Fig. $2 b$ we repeat the experiment with higher level of noise (variance is 0.36 ).

ANCGM which involve inversion. Overall, the computations are comparable. The running time (using Matlab code) in the experiment with variance 0.2 are $t=0.336[\mathrm{sec}]$ for ANCGM and $t=0.1[\mathrm{sec}]$ for our algorithm. For variance 0.36 , it was $t=1.7[\mathrm{sec}]$ for ANCGM and $t=1.19[\mathrm{sec}]$ for ours.

According to Theorem 1, the main flow reaches a steady state when an eigenpair is attained. Thus, it is expected that if $u_{t}=0$ then $\operatorname{sign}(\langle Q(u),-\Delta u\rangle) Q(u) /\|Q(u)\|=$ $-\Delta u /\|\Delta u\|$. In Fig. 3 we plotted $\operatorname{sign}(\langle Q(u),-\Delta u\rangle) Q(u) /\|Q(u)\|$ and $-\Delta u /\|\Delta u\|$. Moreover, on the top left side of these plots we present the approximated eigenvalue $\lambda=\langle-\Delta u, u\rangle /\langle Q(u), u\rangle$ and the computed pointwise ratio $\lambda(x)=$ $-\Delta u(x) / Q(u(x))(u(x)$ is the result of our algorithm at convergence). One can observe these graphs coincide very well. In Fig. 4 the values of $J(u(t))$ and the mean of $u(t)$ as a function of $t$ are shown. Our theoretical analysis predicts $J(u(t))$ should be decreasing with time (Theorem 1 in the time continuous case and the bound on $d t_{M}$ of Eq. (29) in the discrete setting). Moreover, since $u(t)$ is very close to $\mathcal{Q}^{*}$, the mean of $u(t)$ should be almost constant in time (Prop. 1, item 2). It is shown that the numerical implementation approximates well the theoretical analysis.

\subsubsection{Low SNR}

An essential assumption of algorithms based on linearization, such as NCGM, is that the initial guess $u^{0}$ is close enough to the true solution. In this example we try to demonstrate what happens when this assumption is not valid. In this experiment we initialize with the eigenfunction corresponding to $\lambda=2$ and $c=0.25$ and add noise with normal distribution $N \sim \mathcal{N}(0.25,0.25)$. We compared our algorithm and NCGM, giving both of them this initial function (and additionally providing NCGM the correct $\lambda$ ). Fig. 5 shows the results of this experiment. In Fig. 5a the eigenfunction $u^{*}$, the initial function $u^{0}$, our 


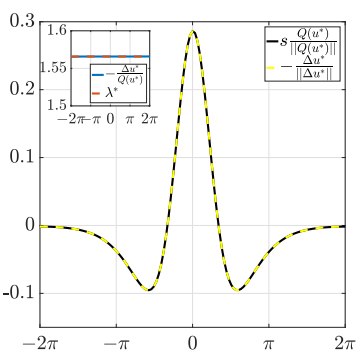

(a) $N \sim \mathcal{N}(0.1,0.2)$

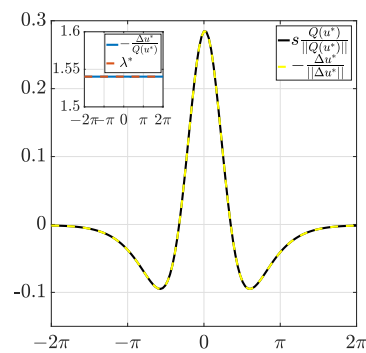

(b) $N \sim \mathcal{N}(0.1,0.36)$

Figure 3: We show here that our algorithm indeed reaches an eigenpair. The results of $T /\|T\|$ and $s Q /\|Q\|$ are shown when the algorithm converges for the $\mathrm{KdV}$ equation. One can observe they coincide very well. The black solid line stands for $s Q(u) /\|Q(u)\|$ and dashed yellow line is $-\Delta u /\|\Delta u\|$. In left top side the approximated $\lambda^{*}=\left\langle-\Delta u^{*}, u^{*}\right\rangle /\left\langle Q\left(u^{*}\right), u^{*}\right\rangle$ is plotted with dashed red line and the solid blue line is the ratio $-\Delta u / Q(u)$.

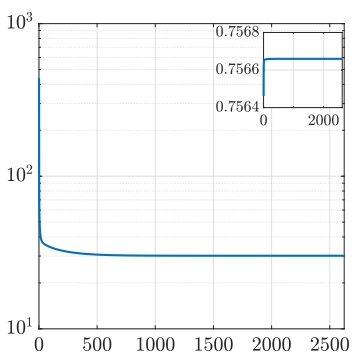

(a) $N \sim \mathcal{N}(0.1,0.2)$

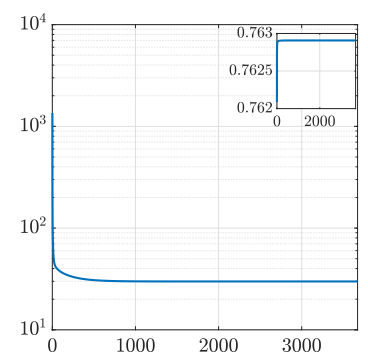

(b) $N \sim \mathcal{N}(0.1,0.36)$

Figure 4: The value of $J\left(u^{k}\right)$ and the mean of $u^{k}$ (right top axis) as a function of iteration number $k$ when applying our flow on KdV for the experiments depicted in Fig. 2. 


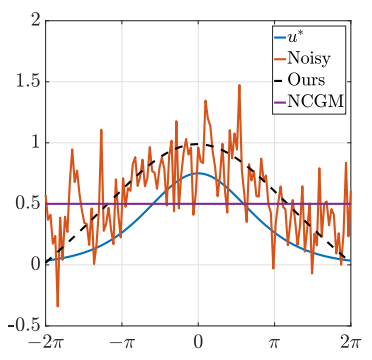

(a) $N \sim \mathcal{N}(0.25,0.25)$

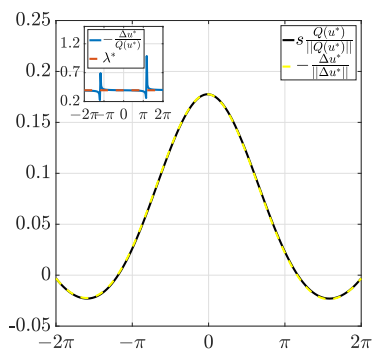

(b) Two opposite counterparts(c) $J(u)$ and mean of $u$ (right of $u_{t}$.

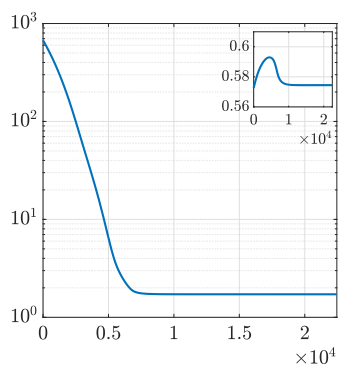

top axis) as a function of the iteration number.

Figure 5: Experiment when the premise $\left\|u^{*}-u^{0}\right\|<<1$ does not hold. Fig. 5a summarizes the result of NCGM and our flow when the initial function is far from the eigenpair (high level of noise). Whereas NCGM collapses to the trivial solution our flow reaches a meaningful solutions. In Fig. 5b it is demonstrated that a good eigenpair approximation is attained. In Fig. 5c the evolution of $J$ and the mean of $u$ as a function of number of iterations are presented.

algorithm and NCGM results are plotted. Our algorithm converges to another eigenfunction whereas NCGM collapses to a trivial solution. Fig. 5b illustrates the similarity of the two opposite normalized counterparts of the flow $u_{t}$, i.e. $s Q(u) /\|Q(u)\|$ and $-\Delta u /\|\Delta u\|$ showing we indeed approximate an eigenfunction well. In Fig. 5 c the value of $J$ and the mean of $u$ are plotted as a function of iteration.

\subsubsection{One Dimensional Nonlinear Schrdinger (NLS) Equation}

In this part we would like to emphasize the simplicity of our approach. Our algorithm is easy to adjust to other applications and the adjustment amounts to redefining $Q(u)$ and its variational derivative. We demonstrate this by the NLS equation as discussed in [35, 43]:

$$
i \frac{\partial u}{\partial t}+\frac{\partial^{2} u}{\partial x^{2}}-k\left|u^{2}\right| u=0
$$

To find a stationary solution we assign $u(x, t)=u(x) e^{i \mu(u) t}$. This yields $-u_{x x}=$ $-\mu(u) u-k u^{3}$, which can be rewritten as $-u_{x x}=\lambda\left(-\hat{\mu}(u) u-\hat{k} u^{3}\right)$ with $\hat{\mu}(u)=$ $1-3 u^{2}$ and $\hat{k}=2$. Finally we reach the problem formulation of (1),

$$
-u_{x x}=\lambda\left(u^{3}-u\right) .
$$

Therefore, we have

$$
Q(u)=u^{3}-u
$$




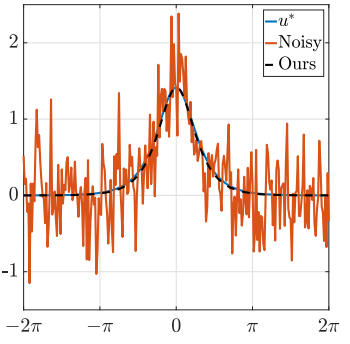

(a) Numerical eigenpair result.

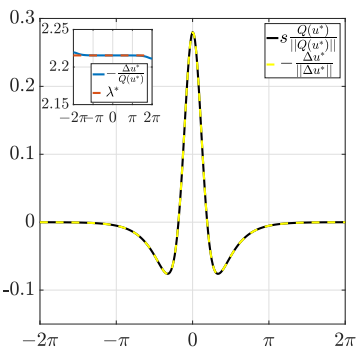

(b) Two counterparts of $u_{t}$.

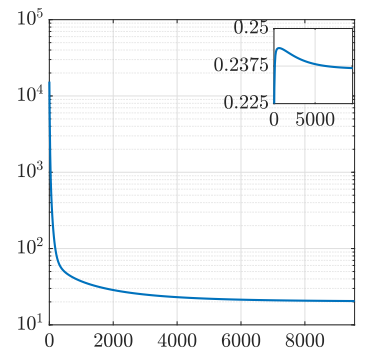

(c) $J$ and the mean of $u$ as a function of iteration number.

Figure 6: One dimension NLS equation. The initial function $u^{0}$ is an eigenfunction with additive noise, $\mu=0, \sigma^{2}=0.45$. Our algorithm result is shown in Fig. 6a. In Fig. 6b the two counterparts of the main flow are shown when an eigenpair is attained. The functional $J$ and the mean of $u$ are presented in Fig. 6c. NCGM diverges in this experiment and ANCGM converges to a trivial solution $(\lambda=0)$. Both of these results are not in the same scale as the initial signal $u^{0}$ and therefore are not presented.

and

$$
\partial Q(u)=3 u^{2}-1 .
$$

We initiate the algorithm with $u^{0}=u^{*}+N$ where $u^{*}$ is the eigenfunction $u(x)=\sqrt{2} \operatorname{sech}(\sqrt{\lambda} x)$ and $N \sim \mathcal{N}(0,0.45)$, see Fig. 6. We similarly initiate the algorithms NCGM and ANCGM. The first diverges and the second one converges to the trivial solution, $\lambda=0$. Both of these results are not in the same scale as the initial signal and therefore are not plotted.

\subsection{Two Dimensions NLS Equation}

The following experiment is based on Example 3.4 from [39] and demonstrates the performance of our algorithm in 2D. We apply our algorithm on NLS equation with periodic potentials which models nonlinear light propagation as well as Bose-Einstein condensate's dynamics in optical lattices. It is formulated as:

$$
i U_{t}+U_{x x}+U_{y y}-V_{0}\left(\sin ^{2} x+\sin ^{2} y\right) U+\sigma|U|^{2} U=0 .
$$

This equation is discussed in details in $[14,28,36]$ and admits a rich variety of solitary waves of the form of $U(x, y, t)=u(x, y) e^{-i \mu t}$, where $u(x, y)$ satisfies the equation:

$$
u_{x x}+u_{y y}-V_{0}\left(\sin ^{2} x+\sin ^{2} y\right) u+\sigma|u|^{2} u=-\mu u .
$$

In our example $\mu=4.11, V 0=6, \sigma=1$ and Dirichlet boundary conditions are used. There is no known analytic solution for these equations. The initial 


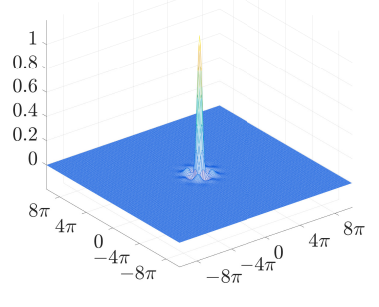

(a) The initial function $u^{0}$

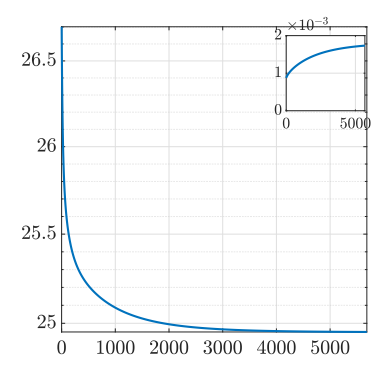

(d) $J(u)$ and mean of $u$ (right top axis)

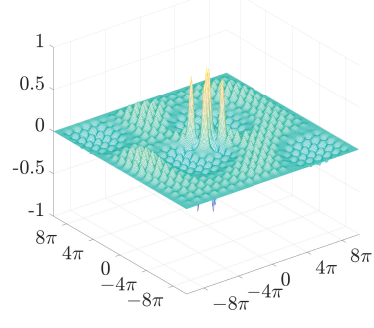

(b) Result of NCGM

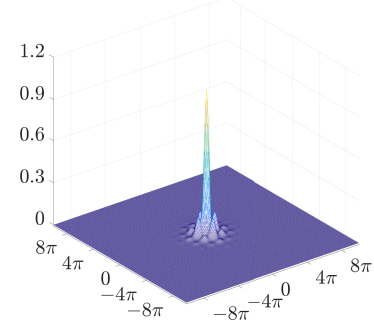

(e) Our flow's result

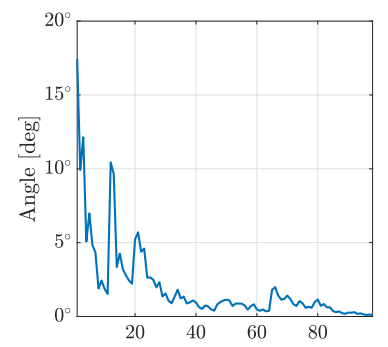

(c) Angle Vs. Iterations, NCGM

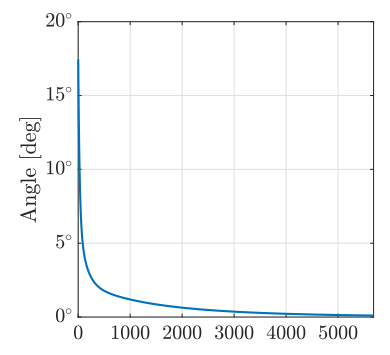

(f) Angle Vs. Iterations, ours

Figure 7: Two dimension NLS.

function of our algorithm is $u_{0}(x, y)=1.15 \operatorname{sech}\left(\sqrt{x^{2}+y^{2}}\right)$. The settings for our flow are:

$$
\begin{aligned}
Q(u) & =-V_{0}\left(\sin ^{2} x+\sin ^{2} y\right) u+\sigma u^{3}+\mu u, \\
\partial Q(u) & =-V_{0}\left(\sin ^{2} x+\sin ^{2} y\right)+3 \sigma u^{2}+\mu .
\end{aligned}
$$

The results of our algorithm and NCGM with $u^{0}$ are summarized in Fig. 7. In Fig. 7a the initial guess $u^{0}$ is shown. The results of NCGM and our flow are presented in Figs. $7 \mathrm{~b}$ and $7 \mathrm{e}$, respectively, and the angle evolutions along iterations are presented in Figs. 7c and 7f. In Fig. 7d we show the behavior of the functional $J$ and the mean of $u$ as a function of time.

We performed a similar experiment of the problem with noise level of $\mathcal{N}(0,0.0125)$. The result of the experiment are similarly organized in Fig. 8. It is easy to see that NCGM converges approximately to the linear case with very small amplitude signal. 


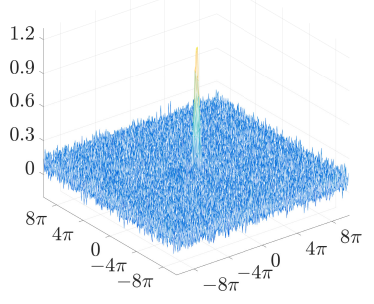

(a) Initial function $u^{0}$

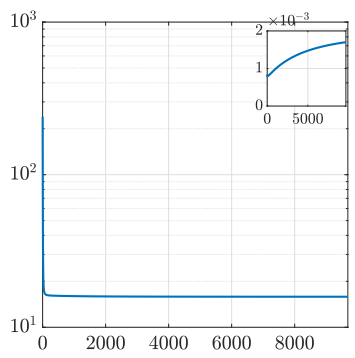

(d) $J(u)$ and mean of $u$ (right top axis)

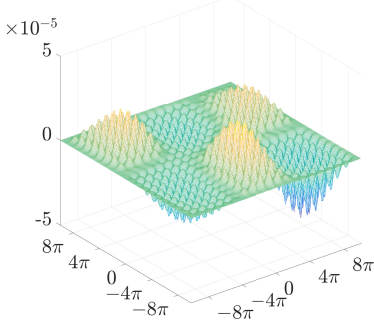

(b) Result of NCGM

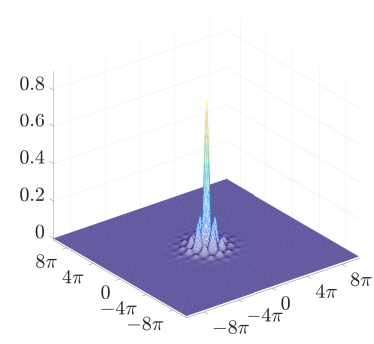

(e) Our flow's result

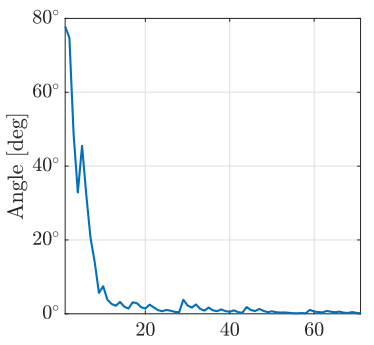

(c) Angle Vs. Iterations, NCGM

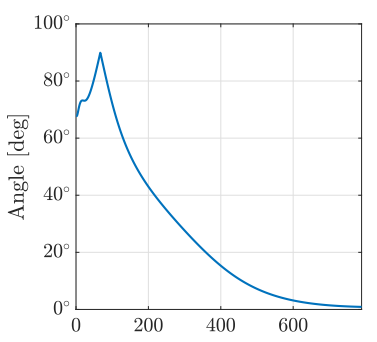

(f) Angle Vs. Iterations, ours

Figure 8: Two dimension NLS with noisy initial function. 

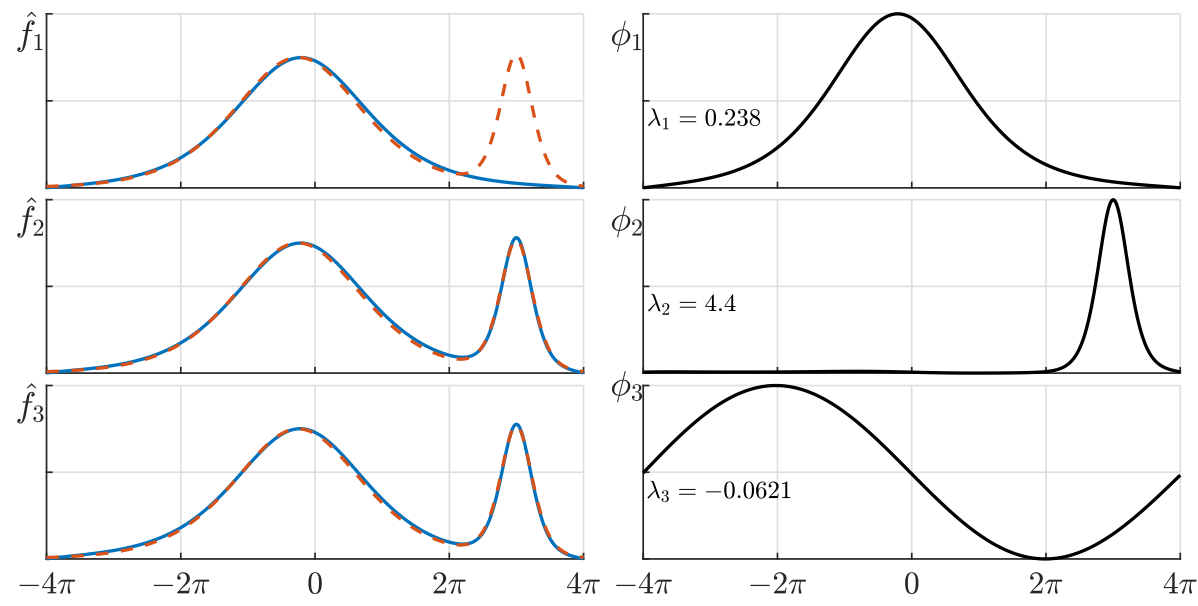

Figure 9: Function decomposition. On the left column, the dashed red line is the initial signal $u_{0}$, which is a combination of two eigenfunctions $(\lambda=0.25, \lambda=$ 4). The blue solid lines are the reconstructions of the signal up to the $k$ th eigenfunction, i.e. $\hat{f}_{k}=\sum_{i=1}^{k} \alpha_{k} \cdot \phi_{k}$ as described in Algo. 3. On the right column, we show the eigenfunctions found by Algo. 3, in the respective order. The first two functions are very similar to the original ones. The third one is a residual function of low amplitude (dominated by the linear part).

\subsection{Decomposition into Eigenfunctions}

A signal may be modeled in some cases by a linear combination of nonlinear eigenfunctions. In this case we would like to decompose it into its basic elements. We suggest below a simple iterative algorithm to perform that (Algo. 3). In Fig. 9 we show an example of such a decomposition for the case of KdV. We compute the eigenfunctions $\phi_{1}, \phi_{2}, \phi_{3}$ with respective eigenvalues $\lambda_{1}, \lambda_{2}, \lambda_{3}$. We used as initial condition a combination of two eigenfunctions of $\mathrm{KdV}$ (with eigenvalues $\lambda=0.25, \lambda=4$ ). One of them is translated in space (therefore their correlation is low). In the decomposition results, the first two eigenfunctions are very similar to the original ones. The third one is of small amplitude. In that case the linear part becomes dominant and the problem reduces to finding eigenfunctions of the ordinary heat equation. Thus, we get a harmonic function.

\section{Conclusions}

In this paper we presented a new nonlinear flow which can generate a very broad family of nonlinear eigenfunctions. The flow reaches a steady state only for eigenpairs. Moreover, the process is a forward flow, which is based on the 


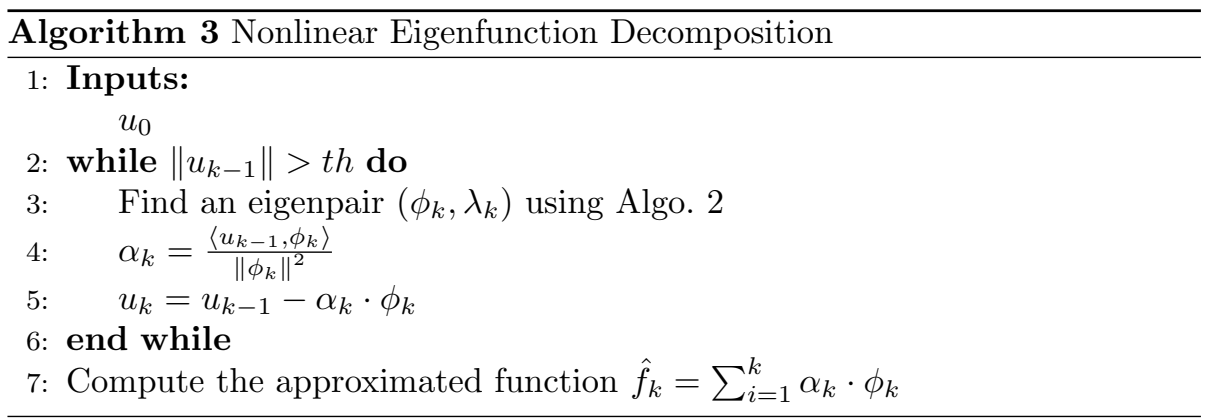

original operators and does not need their inversion. We thus obtain a very stable process and our assumptions regarding the operators are less restrictive. When $T$ is the negative Laplacian operator, we prove that the flow decreases the Dirichlet energy. This yields a highly stable smoothing process, which is robust to noise.

We introduced the space $\mathcal{Q}^{*}$, where all nontrivial eigenfunctions reside in, and explained the requirement of the flow to be constrained to this space. A complementary flow was proposed in order to achieve this requirement. We have tested our algorithm on several nonlinear eigenvalue problems in one and two dimensions, and have shown the stability of the algorithm and its robustness to various initial conditions. Finally, it was demonstrated how potentially our process can be used iteratively, in order to decompose a signal into a linear combination of eigenpairs.

\section{A Considerations for the stopping condition}

Implementations of numerical solutions are concerned with issues related to the appropriate stopping condition. As $T(u)$ and $Q(u)$ are fully correlated when eigensolution of Eq. (1) is attained, the most natural criteria is the angle between them (see Eq. (16)). Other criteria worth mentioning are $(d / d t) J(u)$ Eq. (8) and $\|M(u)\|$ which are shown to be zero when an eigenpair is reached. The norm of the main flow can be calculated as:

$$
\|M(u)\|=\sqrt{2} \sqrt{1-\frac{|\langle Q(u), T(u)\rangle|}{\|Q(u)\| \cdot\|T(u)\|}} .
$$

If $u \notin \mathcal{Q}^{*}$ then $\exists e \in \mathcal{B}$ s.t. $\langle Q(u), e\rangle \neq 0$. Let us denote the projection of $Q(u)$ on $\mathcal{T}^{\perp}$ as:

$$
Q^{\perp}(u)=\sum_{e_{i} \in \mathcal{B}} e_{i} \cdot\left\langle Q(u), e_{i}\right\rangle \neq 0,
$$

and the "residue" of $Q(u)$ as:

$$
Q^{\|}(u)=Q(u)-Q^{\perp}(u) .
$$


As $E(u)$ is positive we get the following inequalities:

$$
\begin{aligned}
|\cos (\theta)| & =\frac{|\langle T(u), Q(u)\rangle|}{\|T(u)\| \cdot\|Q(u)\|}=\frac{\left|\left\langle T(u), Q^{\|}(u)\right\rangle\right|}{\|T(u)\| \cdot\|Q(u)\|} \\
& \leq \frac{\left\|Q^{\|}(u)\right\|}{\|Q(u)\|}=\frac{\left\|Q^{\|}(u)\right\|}{\sqrt{\left\|Q^{\|}(u)\right\|^{2}+2 E(u)}}
\end{aligned}
$$

and similarly

$$
\begin{gathered}
\|M(u)\|=\sqrt{2} \sqrt{1-\frac{|\langle Q(u), T(u)\rangle|}{\|Q(u)\| \cdot\|T(u)\|}} \\
\geq \sqrt{2} \sqrt{1-\frac{\left\|Q^{\|}(u)\right\|}{\sqrt{\left\|Q^{\|}(u)\right\|^{2}+2 E(u)}}}, \\
\frac{d}{d t} J(u) \leq\left(\frac{\left\|Q^{\|}(u)\right\|}{\sqrt{\left\|Q^{\|}(u)\right\|^{2}+2 E(u)}}-1\right)\|T(u)\| .
\end{gathered}
$$

As long as $E(u)>0$ our analysis has shown that a precise eigenfunction cannot be obtained. However, one can set a small threshold on $E(u)$, for example, if $E(u) \leq \delta$ we have:

$$
\begin{aligned}
\|M(u)\|_{t h} & =\sqrt{2} \sqrt{1-\frac{\left\|Q^{\|}(u)\right\|}{\sqrt{\left\|Q^{\|}(u)\right\|^{2}+4 \delta}}} \\
\frac{d}{d t} J(u)_{t h} & =\left(\frac{\left\|Q^{\|}(u)\right\|}{\sqrt{\left\|Q^{\|}(u)\right\|^{2}+4 \delta}}-1\right)\|T(u)\| \\
|\cos (\theta)|_{t h} & =\frac{\left\|Q^{\|}(u)\right\|}{\sqrt{\left\|Q^{\|}(u)\right\|^{2}+4 \delta}} .
\end{aligned}
$$

We note that the above discussion concerns the true evolution in the time continuous setting. However its implications can naturally affect the numerical algorithms.

\section{References}

[1] J. Álvarez and A. Durán. Petviashvili type methods for traveling wave computations: I. analysis of convergence. Journal of Computational and Applied Mathematics, 266:39-51, 2014.

[2] J. Álvarez and A. Durán. Numerical generation of periodic traveling wave solutions of some nonlinear dispersive wave systems. Journal of Computational and Applied Mathematics, 316:29-39, 2017. 
[3] F. Andreu, C. Ballester, V. Caselles, and J. M. Mazón. Minimizing total variation flow. Differential and Integral Equations, 14(3):321-360, 2001.

[4] V. Apidopoulos, J.-F. Aujol, and C. Dossal. On a second order differential inclusion modeling the fista algorithm. 2017.

[5] J.-F. Aujol, G. Gilboa, and N. Papadakis. Theoretical analysis of flows estimating eigenfunctions of one-homogeneous functionals for segmentation and clustering. 2017.

[6] W. Bao and Q. Du. Computing the ground state solution of bose-einstein condensates by a normalized gradient flow. SIAM Journal on Scientific Computing, 25(5):1674-1697, 2004.

[7] W. Bao and W. Tang. Ground-state solution of bose-einstein condensate by directly minimizing the energy functional. Journal of Computational Physics, 187(1):230-254, 2003.

[8] M. Benning and M. Burger. Ground states and singular vectors of convex variational regularization methods. Methods and Applications of Analysis, 20(4):295-334, 2013.

[9] H. Brezis. Ope rateurs maximaux monotones et semi-groupes de contractions dans les espaces de Hilbert, volume 5. Elsevier, 1973.

[10] M. Burger, G. Gilboa, M. Moeller, L. Eckardt, and D. Cremers. Spectral decompositions using one-homogeneous functionals. SIAM Journal on Imaging Sciences, 9(3):1374-1408, 2016.

[11] M. Burger and S. Osher. A guide to the tv zoo. In Level set and PDE based reconstruction methods in imaging, pages 1-70. Springer, 2013.

[12] M. Caliari, A. Ostermann, S. Rainer, and M. Thalhammer. A minimisation approach for computing the ground state of gross-pitaevskii systems. Journal of Computational Physics, 228(2):349-360, 2009.

[13] A. Chambolle, V. Caselles, D. Cremers, M. Novaga, and T. Pock. An introduction to total variation for image analysis. Theoretical foundations and numerical methods for sparse recovery, 9(263-340):227, 2010.

[14] F. Dalfovo, S. Giorgini, L. P. Pitaevskii, and S. Stringari. Theory of bose-einstein condensation in trapped gases. Reviews of Modern Physics, 71(3):463, 1999.

[15] V. Demyanov and G. S. Tamasyan. Exact penalty functions in isoperimetric problems. Optimization, 60(1-2):153-177, 2011.

[16] V. F. Dem'yanov. Exact penalty functions and problems of variation calculus. Automation and Remote Control, 65(2):280-290, 2004. 
[17] C. Effenberger. Robust solution methods for nonlinear eigenvalue problems. $\mathrm{PhD}$ thesis, PhD thesis, EPF Lausanne, 2013.

[18] I. Ekeland and R. Temam. Convex analysis and variational problems, volume 28. Siam, 1999.

[19] J. J. García-Ripoll and V. M. Pérez-García. Optimizing schrödinger functionals using sobolev gradients: Applications to quantum mechanics and nonlinear optics. SIAM Journal on Scientific Computing, 23(4):1316-1334, 2001.

[20] G. Gilboa. A spectral approach to total variation. In International Conference on Scale Space and Variational Methods in Computer Vision, pages 36-47. Springer, 2013.

[21] G. Gilboa. A total variation spectral framework for scale and texture analysis. SIAM journal on Imaging Sciences, 7(4):1937-1961, 2014.

[22] S. Güttel and F. Tisseur. The nonlinear eigenvalue problem. Acta Numerica, 26:1-94, 2017.

[23] M. Hein and T. Bühler. An inverse power method for nonlinear eigenproblems with applications in 1-spectral clustering and sparse pca. In Advances in Neural Information Processing Systems, pages 847-855, 2010.

[24] J. HORÁK. Numerical investigation of the smallest eigenvalues of the p-laplace operator on planar domains. Electronic Journal of Differential Equations, 2011(132):1-30, 2011.

[25] T. I. Lakoba. Conjugate gradient method for finding fundamental solitary waves. Physica D: Nonlinear Phenomena, 238(23-24):2308-2330, 2009.

[26] T. I. Lakoba and J. Yang. A generalized petviashvili iteration method for scalar and vector hamiltonian equations with arbitrary form of nonlinearity. Journal of Computational Physics, 226(2):1668-1692, 2007.

[27] Y. Meyer. Oscillating Patterns in Image Processing and Nonlinear Evolution Equations: The Fifteenth Dean Jacqueline B. Lewis Memorial Lectures. American Mathematical Society, Boston, MA, USA, 2001.

[28] O. Morsch and M. Oberthaler. Dynamics of bose-einstein condensates in optical lattices. Reviews of modern physics, 78(1):179, 2006.

[29] R. Z. Nossek and G. Gilboa. Flows generating nonlinear eigenfunctions. Journal of Scientific Computing, pages 1-30, 2016.

[30] J. A. Pava. Nonlinear dispersive equations: existence and stability of solitary and periodic travelling wave solutions. Number 156. American Mathematical Soc., 2009. 
[31] D. E. Pelinovsky and Y. A. Stepanyants. Convergence of petviashvili's iteration method for numerical approximation of stationary solutions of nonlinear wave equations. SIAM Journal on Numerical Analysis, 42(3):11101127, 2004.

[32] V. Petviashvili and O. Pokhotelov. Solitary waves in plasmas and in the atmosphere, gordon and breach pub. Co., Reding, 1992.

[33] V. I. Petviashvili. Equation of an extraordinary soliton. Fizika plazmy, 2:469-472, 1976.

[34] L. I. Rudin, S. Osher, and E. Fatemi. Nonlinear total variation based noise removal algorithms. Physica D: nonlinear phenomena, 60(1-4):259-268, 1992.

[35] A. Shabat and V. Zakharov. Exact theory of two-dimensional self-focusing and one-dimensional self-modulation of waves in nonlinear media. Soviet physics JETP, 34(1):62, 1972.

[36] M. Skorobogatiy and J. Yang. Fundamentals of photonic crystal guiding. Cambridge University Press, 2009.

[37] L. N. Trefethen and D. Bau III. Numerical linear algebra, volume 50. Siam, 1997.

[38] C. Wang and J. Zhou. A new approach for numerically solving nonlinear eigensolution problems. Journal of Scientific Computing, 64(1):109-129, 2015 .

[39] J. Yang. Newton-conjugate-gradient methods for solitary wave computations. Journal of Computational Physics, 228(18):7007-7024, 2009.

[40] J. Yang and T. I. Lakoba. Universally-convergent squared-operator iteration methods for solitary waves in general nonlinear wave equations. Studies in Applied Mathematics, 118(2):153-197, 2007.

[41] X. Yao and J. Zhou. Numerical methods for computing nonlinear eigenpairs: Part i. iso-homogeneous cases. SIAM Journal on Scientific Computing, 29(4):1355-1374, 2007.

[42] N. J. Zabusky and M. D. Kruskal. Interaction of" solitons" in a collisionless plasma and the recurrence of initial states. Physical review letters, 15(6):240, 1965.

[43] V. E. Zakharov and S. V. Manakov. On the complete integrability of a nonlinear schrödinger equation. Theoretical and Mathematical Physics, 19(3):551-559, 1974. 